\title{
MicroRNA-502-3p promotes Mycobacterium tuberculosis survival in macrophages by modulating the inflammatory response by targeting ROCK1
}

\author{
FANG LIU ${ }^{1}$, ZHEN DONG $^{2}$, YUEFU LIN ${ }^{3}$, HAIBO YANG $^{4}$, \\ PINGPING WANG ${ }^{5}$ and YONGXIA ZHANG ${ }^{6}$
}

\author{
${ }^{1}$ Respiratory Endoscopy Room; ${ }^{2}$ East Medical District Office; ${ }^{3}$ Department of Prevention and \\ ${ }^{4}$ Department of Occupational Diseases, Linyi People's Hospital; ${ }^{5}$ Rehabilitation Department, \\ Shandong Coal Linyi Hot Spring Sanatorium; ${ }^{6}$ Emergency Department, \\ Linyi People's Hospital, Linyi, Shandong 276034, P.R. China
}

Received December 21, 2020; Accepted July 29, 2021

DOI: $10.3892 / \mathrm{mmr} .2021 .12393$

\begin{abstract}
Tuberculosis (TB) is caused by Mycobacterium tuberculosis (M. tuberculosis) infection and has the highest mortality rate of any single infectious disease worldwide. The aim of the present study was to investigate the function of microRNA (miR)-502-3p in M. tuberculosis-infected macrophages. The Gene Expression Omnibus database was used to analyze miR-502-3p expression in patients with TB and healthy individuals. THP-1 and RAW 264.7 cells were transfected with miR-502-3p mimic, miR-502-3p inhibitor, pcDNA3.1-ROCK1 or their negative controls. The expression levels of miR-502-3p and inflammatory cytokines were evaluated using reverse transcription-quantitative PCR. The colony-forming unit assay was performed to assess the survival of $M$. tuberculosis in macrophages, and Toll-like receptor (TLR)4/NF- $\kappa \mathrm{B}$ signaling pathway-associated protein expression levels were detected by western blotting. The nuclear translocation of $\mathrm{NF}-\kappa \mathrm{B}$ p 65 was detected via immunocytochemistry. TargetScan was used to predict the binding sites between miR-502-3p and ROCK1. The interaction between miR-502-3p and Rho-associated coiled-coil-forming protein kinase 1 (ROCK1) was confirmed using a dual-luciferase reporter assay; ROCK1 was demonstrated to be a direct target gene of miR-502-3p. Results from the present study demonstrated that miR-502-3p expression was significantly increased during $M$. tuberculosis infection in macrophages. Upregulation of miR-502-3p expression levels significantly
\end{abstract}

Correspondence to: Dr Yongxia Zhang, Emergency Department, Linyi People's Hospital, 233 Phoenix Street, Linyi, Shandong 276034, P.R. China

E-mail: yongxia952@126.com

Key words: Mycobacterium tuberculosis, inflammation, macrophages, microRNA-502-3p, Rho-associated coiled-coil-forming protein kinase 1 enhanced the survival of intracellular M. tuberculosis. IL-6, TNF- $\alpha$, and IL-1 $\beta$ mRNA expression levels were significantly upregulated during $M$. tuberculosis infection but were downregulated by miR-502-3p overexpression. Moreover, miR-502-3p mimics transfection significantly downregulated TLR4/NF- $\kappa \mathrm{B}$ signaling pathway-associated protein expression and significantly reduced nuclear transcription of $\mathrm{NF}-\kappa \mathrm{B}$ in $M$. tuberculosis-infected macrophages. ROCK1 overexpression reversed the miR-502-3p inhibitory effect on cytokine production in $M$. tuberculosis-infected macrophages. In conclusion, miR-502-3p/ROCK1 may serve an anti-inflammatory role and may improve the survival of M.tuberculosis within macrophages, which may provide a promising therapeutic target for TB.

\section{Introduction}

Tuberculosis (TB) is caused by the etiological agent Mycobacterium (M.), which primarily affects the lungs (1). TB was responsible for $\sim 1.5$ million deaths in 2018 worldwide (2). M. tuberculosis parasitizes the macrophages of the host; it manipulates the host's defenses and consequently the immune response (3). Furthermore, $M$. tuberculosis can evade innate immunity to survive and replicate inside macrophages (4). Therefore, the development of therapeutics that prevent immune evasion is crucial.

MicroRNAs (miRNAs/miRs) are a class of non-coding RNAs ( 22 nucleotides long) that serve a role in silencing target gene expression and are associated with immune signaling pathways $(5,6)$. For example, miR-325-3p promotes $M$. tuberculosis survival by targeting ligand of numb-protein $\mathrm{X} 1$ and increasing the phosphorylation of STAT3 (7). Overexpression of miR-26a was reported to modulate the survival of $M$.tuberculosis in macrophages (8). miR-125a inactivates $\mathrm{NF}-\kappa \mathrm{B}$ signaling by targeting TNF receptor associated factor 6 , attenuating inflammation and facilitating the survival of M. tuberculosis (9). Although the potential role of several miRNAs in M. tuberculosis infection has been examined, this field requires further investigation. 
Rho-associated coiled-coil-forming protein kinase 1 (ROCK1) is a downstream effector of RhoA; it acts as a 'molecular switch' in the activation of the monocyte pro-inflammatory response (10). Suppression of ROCK1 expression prevents $\mathrm{NF}-\kappa \mathrm{B}$ signaling in a variety of inflammatory diseases, such as cervical cancer, and is a hallmark of obstructive sleep apnea syndrome $(11,12)$. A previous study has shown that Toll-like receptor (TLR) 4 is involved in the regulation of pulmonary immune responses and recognition of M. tuberculosis (13). $\mathrm{NF}-\kappa \mathrm{B}$ is a downstream effector of the TLR4 signaling pathway and an important pro-inflammatory factor (14). Numerous inflammatory cytokines, including TNF- $\alpha$, IL-1 $\beta$ and IL-6, regulate the TLR4/NF- $\kappa$ B pathway (15).

In the present study, upregulated miRNAs in patients with TB were identified using the Gene Expression Omnibus (GEO) datasets, GSE34608 and GSE116542. miR-502-3p was selected for further studies. The aim of the present study was to investigate the function of miR-502-3p in M. tuberculosis-infected macrophages.

\section{Materials and methods}

Bioinformatics. The GEO (https:/www.ncbi.nlm.nih.gov/geo) database [GSE34608 (PMID: 22547807) and GSE116542] was used to analyze miR-502-3p expression in patients with TB and healthy individuals. In GSE116542, 11 patients with TB ( 8 men; 3 women; age range, $17-51$ years) and 8 healthy individuals (4 men; 4 women; age range, 29-60 years) were collected for exosomal miRNAs extraction. TargetScan 7.2 (http://www.targetscan.org) was used to predict the binding sites between miR-502-3p and ROCK1.

Cell culture. The human leukemia monocytic THP-1 and the mouse macrophage-like RAW 264.7 cell lines were purchased from The Cell Bank of Type Culture Collection of The Chinese Academy of Sciences. Cells were maintained in an incubator $\left(37^{\circ} \mathrm{C} ; 5 \% \mathrm{CO}_{2} ; 70 \%\right.$ relative humidity) in RPMI-1640 medium containing $10 \%$ fetal bovine serum (Hyclone; Cytiva).

Transfection. THP-1 and RAW 264.7 cells were plated on 12-well plates at a seeding density of $3 \times 10^{5}$ cells/well and transfected with miR-502-3p mimic, miR-502-3p inhibitor, pcDNA3.1-ROCK1 (Shanghai GenePharma Co., Ltd.), or their negative controls (NCs), mimic NC, inhibitor NC and pcDNA3.1-NC (50 pg/well) at $37^{\circ} \mathrm{C}$ for $24 \mathrm{~h}$, using Lipofectamine ${ }^{\circledR} 3000$ (Invitrogen; Thermo Fisher Scientific, Inc.). At $24 \mathrm{~h}$ following transfection, the transfected macrophages were used for other experimental assays. The sequences are listed in Table I.

Infection. M. tuberculosis strain H37Rv (cat. no. 25618; AmericanTypeCultureCollection) wascultured in Middlebrook 7H9 broth media (Beijing Solarbio Science \& Technology Co., Ltd.) containing $10 \%$ oleic acid albumin dextrose catalase enrichment (OADC; BD Biosciences) at $37^{\circ} \mathrm{C}$. Transfected THP-1 cells were incubated with $100 \mathrm{nM}$ phorbol 12-myristate 13-acetate (PMA; Sigma-Aldrich; Merck KGaA) at $37^{\circ} \mathrm{C}$ for $48 \mathrm{~h}$ until differentiation into human macrophages occurred. Following transfection and PMA-differentiation, THP-1 cells and RAW 264.7 cells $\left(5.0 \times 10^{5}\right)$ were infected with the M. tuberculosis strain H37Rv at a multiplicity of infection (MOI) of $1,2,5$ and 10 at $37^{\circ} \mathrm{C}$ for $48 \mathrm{~h}$. In subsequent experiments, cells were infected with $M$. tuberculosis $\mathrm{H} 37 \mathrm{Rv}$ at $37^{\circ} \mathrm{C}$ for $3,6,12,24$ and $48 \mathrm{~h}$ at an MOI of 10.

Reverse transcription-quantitative PCR (RT-qPCR). Total RNA was extracted from the transfected and infected THP-1 and RAW 264.7 cells using TRIzol ${ }^{\circledR}$ (Invitrogen; Thermo Fisher Scientific, Inc.) according to the manufacturer's protocol. Total RNA $(1 \mu \mathrm{g})$ was reverse transcribed into cDNA using the M-MLV First Strand Kit (Invitrogen; Thermo Fisher Scientific, Inc.) according to the manufacturer's protocol. qPCR was subsequently performed using the SYBR-Green PCR master mix (Applied Biosystems; Thermo Fisher Scientific, Inc.) in a 7900HT Fast Real-Time PCR System (Applied Biosystems; Thermo Fisher Scientific, Inc.). qPCR was conducted under the following conditions: Initial denaturation at $95^{\circ} \mathrm{C}$ for $20 \mathrm{sec}$, followed by 40 cycles of $95^{\circ} \mathrm{C}$ for $5 \mathrm{sec}, 60^{\circ} \mathrm{C}$ for $30 \mathrm{sec}$ and $72^{\circ} \mathrm{C}$ for $15 \mathrm{sec}$. The primers used for qPCR are provided in Table II. For IL-6, TNF- $\alpha$ and IL-1 $\beta$, $\beta$-actin was used as the internal reference gene. Bulge-loop ${ }^{\mathrm{TM}}$ miRNA RT-qPCR primer sets specific for miR-502-3p were designed by Guangzhou RiboBio Co., Ltd. For miR-502-3p, U6 was used as the internal control. Relative expression levels were analyzed using the $2^{-\Delta \Delta \mathrm{Cq}}$ method (16).

Colony-forming unit (CFU) assay. Following transfection, cells ( $3 \times 10^{5}$ cells/well) were infected with $M$.tuberculosis $(\mathrm{MOI}=10)$ for $48 \mathrm{~h}$ and lysed with $0.5 \%$ Triton $\mathrm{X}-100$ at $25^{\circ} \mathrm{C}$ for $20 \mathrm{~min}$. A 10 -fold serial dilution method was used for quantitative culture of bacterial colonies. The cell lysate was diluted with Middlebrook 7H9 broth media and added to Middlebrook 7H10 agar plates (Beijing Solarbio Science \& Technology Co., Ltd.) supplemented with $10 \%$ OADC. Plates were incubated at $37^{\circ} \mathrm{C}$ for 3 weeks before the colonies were quantified manually. A colony referred to the growth of bacteria on solid medium that could be identified by the naked eye.

Dual-luciferase reporter assay. TargetScan was used to predict the mRNAs that may have a target site of miR-502-3p. Wild-type (WT) and mutant (MUT) ROCK1 3'-untranslated regions (UTRs) were cloned into the firefly luciferase reporter plasmid psi-CHECK2 (Qiagen China Co., Ltd.) to synthesize the ROCK1-WT and ROCK1-Mut reporter plasmids. 293T cells (American Type Culture Collection) at $5 \times 10^{4}$ cells/well in 24-well plates were co-transfected with WT or Mut ROCK1 3'-UTR reporter plasmids and miR-502-3p mimic or mimic NC using Lipofectamine ${ }^{\circledR} 3000$ (Invitrogen; Thermo Fisher Scientific, Inc.) at $37^{\circ} \mathrm{C}$. After $48 \mathrm{~h}$ transfection, luciferase activity was detected using a Dual-Luciferase Reporter Assay System (Promega Corporation), according to the manufacturer's protocol. Renilla luciferase activity was used for normalization.

Western blotting. Total protein was extracted from THP-1 and RAW 264.7 cells $\left(5 \times 10^{5}\right.$ cells/well) using RIPA lysis buffer (Beyotime Institute of Biotechnology). The protein content was assessed using the BCA method. Total protein (50 $\mu \mathrm{g}$ protein/lane) was separated by SDS-PAGE on a $12 \%$ gel. The separated proteins were transferred onto a nitrocellulose membrane (Invitrogen; Thermo Fisher Scientific, Inc.). The membranes were blocked using 5\% skimmed milk for $2 \mathrm{~h}$ at $25^{\circ} \mathrm{C}$. The membranes were incubated with 
Table I. Primer sequences used in transfection.

\begin{tabular}{lc}
\hline Gene & \multicolumn{1}{c}{ Primer sequence $\left(5^{\prime} \rightarrow 3^{\prime}\right)$} \\
\hline $\begin{array}{l}\text { miR-502-3p } \\
\text { mimic }\end{array}$ & AAUGCACCUGGGCAAGGAUUCA \\
Mimic NC & UCACAACCUCCUAGAAAGAGUAGA \\
miR-502-3p & UGAAUCCUUGCCCAGGUGCAUU \\
inhibitor & \\
Inhibitor NC & UCUACUCUUUCUAGGAGGUUGUGA
\end{tabular}

miR, microRNA; NC, negative control.

primary antibodies against the following: ROCK1 $(1: 1,000$; cat. no. 4035), TLR4 (1:1,000; cat. no. 14358; Cell Signaling Technology, Inc.; cat. no. ab13556, Abcam), phosphorylated (p)-p65 (1:1,000; cat. no. 3033; Cell Signaling Technology, Inc.), p65 (1:1,000; cat. no. 8242; Cell Signaling Technology, Inc.), p-IкB $\alpha$ (1:1,000; cat. no. 2859; Cell Signaling Technology, Inc.), I $\kappa \mathrm{B} \alpha(1,000$; cat. no. 4812; Cell Signaling Technology, Inc.) and $\beta$-actin (1:2,000; cat. no. 4970; Cell Signaling Technology, Inc.) overnight at $4^{\circ} \mathrm{C}$. Following three washes of 5 min each with TBS- $0.1 \%$ Tween-20, the membranes were incubated with HRP-conjugated anti-rabbit secondary antibodies $(1: 3,000$; cat. no. A0208; Beyotime Institute of Biotechnology) at $25^{\circ} \mathrm{C}$ for $1 \mathrm{~h}$. Protein bands were visualized using an ECL reagent (Beyotime Institute of Biotechnology) and a gel imaging system (Tanon Science \& Technology Co., Ltd.). Protein bands were semi-quantified using ImageJ software v1.8.0 (National Institutes of Health). $\beta$-actin was used as the loading control.

Immunocytochemistry. THP-1 and RAW 264.7 cells $\left(5 \times 10^{4}\right)$ were fixed in 24-well plates using $4 \%$ paraformaldehyde at $25^{\circ} \mathrm{C}$ for $30 \mathrm{~min}$ (Beyotime Institute of Biotechnology), permeabilized using $0.3 \%$ Triton $\mathrm{X}-100$ at $25^{\circ} \mathrm{C}$ for $20 \mathrm{~min}$ and blocked with $5 \%$ goat serum (Gibco; Thermo Fisher Scientific, Inc.) at $25^{\circ} \mathrm{C}$ for $30 \mathrm{~min}$. Cells were incubated with primary antibody against p-p65 (1:1,600; cat. no. 3033; Cell Signaling Technology, Inc.) overnight at $4^{\circ} \mathrm{C}$. Cells were subsequently incubated with Alexa Fluor 594-conjugated goat anti-rabbit IgG secondary antibody (1:500; cat. no. ab150080; Abcam) for $1 \mathrm{~h}$ in the dark at room temperature. Cell nuclei were stained using DAPI (Beyotime Institute of Biotechnology) at $25^{\circ} \mathrm{C}$ for $30 \mathrm{~min}$. Images were captured using a BX51 fluorescence microscope (Olympus Corporation).

Statistical analysis. All presented data were obtained from at least three independent experiments. Data are shown as the mean \pm SD. Statistical comparisons between two groups were determined by Student's unpaired t-test, whereas comparisons between multiple groups were determined using one-way ANOVA followed by Tukey's post-hoc test. $\mathrm{P}<0.05$ was considered to indicate a statistically significant difference.

\section{Results}

M. tuberculosis infection induces miR-502-3p expression in patients with TB. miRNAs with markedly high expression
Table II. Sequences of primers used for reverse transcription-quantitative PCR.

\begin{tabular}{|c|c|}
\hline Gene & Primer sequence $\left(5^{\prime} \rightarrow 3^{\prime}\right)$ \\
\hline \multirow[t]{2}{*}{$\operatorname{miR}-502-3 p$} & $\begin{aligned} \text { F: } & \text { ACACTCCAGCTGGGAATGCACCT } \\
& \text { GGGCAAGG }\end{aligned}$ \\
\hline & R: CTCAACTGGTGTCGTGGA \\
\hline \multirow[t]{2}{*}{ U6 } & F: CTCGCTTCGGCAGCACA \\
\hline & R: AACGCTTCACGAATTTGCGT \\
\hline \multirow[t]{2}{*}{ IL-6 (human) } & F: ACAACCACGGCCTTCCCTACT \\
\hline & R: CACGATTTCCCAGAGAACATGTG \\
\hline \multirow[t]{2}{*}{ IL-6 (mouse) } & F: GGGCTGCGATGGAGTCAGAG \\
\hline & R: TCCCTCACACAGGGCTCGAC \\
\hline \multirow[t]{2}{*}{ TNF- $\alpha$ (human) } & F: GCCTCTTCTCATTCCTGCTTG \\
\hline & R: GGCCATTTGGGAACTTCTCA \\
\hline \multirow[t]{2}{*}{ TNF- $\alpha$ (mouse) } & F: TGACCCCCATTACTCTGACC \\
\hline & R: TTCAGCGTCTCGTGTGTTTC \\
\hline \multirow[t]{2}{*}{ IL-1 $\beta$ (human) } & F: GTGGCAATGAGGATGACTTGTTC \\
\hline & R: GGTGGTCGGAGATTCGTAGCT \\
\hline \multirow[t]{2}{*}{ IL-1 $\beta$ (mouse) } & F: GAGCAACAAGTGGTGTTCTCC \\
\hline & R: AACACGCAGGACAGGTACAG \\
\hline \multirow[t]{2}{*}{$\beta$-actin (human) } & F: CATGTACGTTGCTATCCAGGC \\
\hline & R: CTCCTTAATGTCACGCACGAT \\
\hline \multirow[t]{2}{*}{$\beta$-actin (mouse) } & F: GTGTGGGCATTTGATGAGCC \\
\hline & R: AGGTCACTTACCTGGTGCCT \\
\hline
\end{tabular}

F, forward; R, reverse; miR, microRNA.

levels in TB compared with the healthy control group were selected by analyzing the GSE34608 and GSE116542 datasets from the GEO database. A total of seven miRNAs were identified as being the same between the datasets (Fig. 1A). The current study aimed to investigated ROCK1 in TB. TargetScan predicted the binding sites between miR-502-3p and ROCK1. Thus, miR-502-3p was chosen from the seven miRNAs. miR-502-3p expression levels were significantly elevated in patients with TB compared with healthy individuals in the GSE116542 database (Fig. 1B). miR-502-3p expression levels in $M$. tuberculosis-infected macrophages increased in a time and dose-dependent manner (Fig. 1C and D). The expression level of miR-502-3p in macrophages at $48 \mathrm{~h}$ post-infection was $>3$-fold higher than that in uninfected control (Fig. 1C). Moreover, there was a $\sim 3$-fold increase in miR-502-3p expression in macrophages cells at a MOI of 10 for $48 \mathrm{~h}$ compared with uninfected cells (Fig. 1D). Macrophages infected with M. tuberculosis at an MOI of 10 for $48 \mathrm{~h}$ were used for subsequent experiments.

miR-502-3p facilitates $M$. tuberculosis survival in macrophages. To further determine the potential role of miR-502-3p in the cellular immune response during M. tuberculosis infection, THP-1 and RAW 264.7 cells were transfected with either miR-502-3p mimic or inhibitor. miR-502-3p expression levels significantly increased following miR-502-3p mimic transfection compared with mimic NC, and significantly decreased following miR-502-3p inhibitor 
A

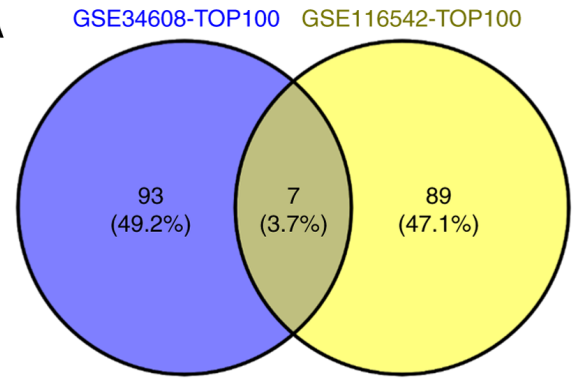

C

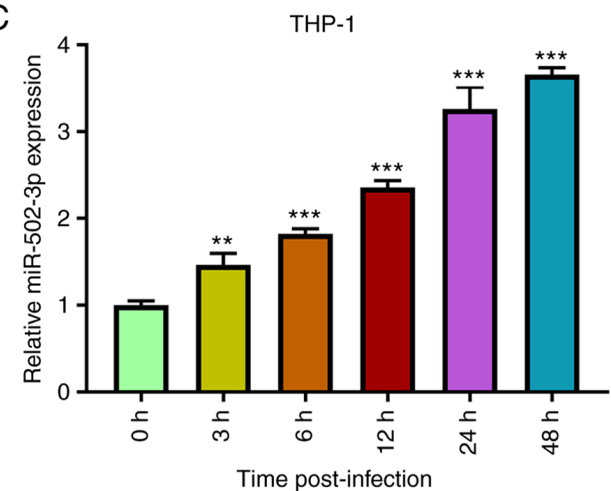

D

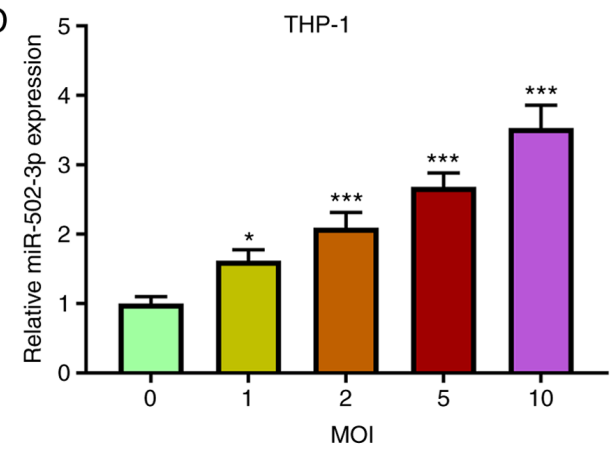

B
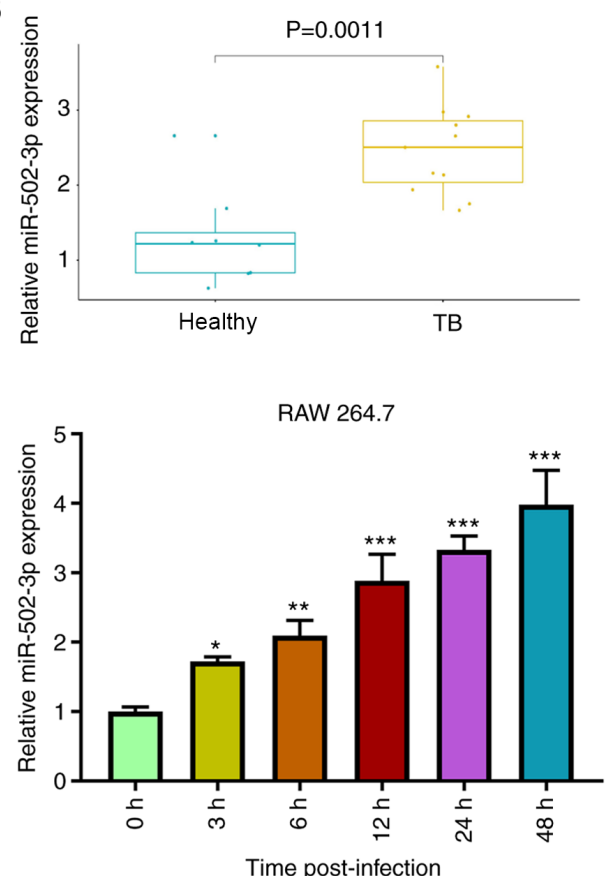

RAW 264.7

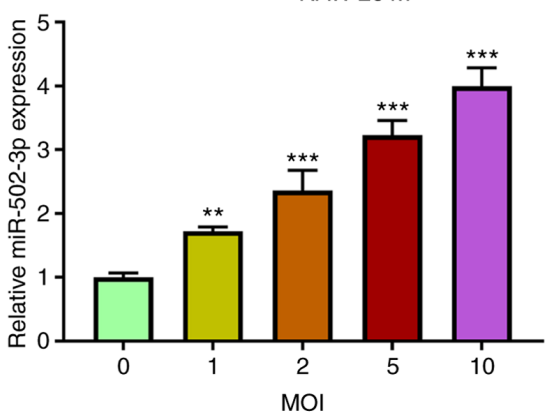

Figure 1. M.tuberculosis infection induces miR-502-3p expression in patients with TB. (A) Seven miRNAs overlapped between the GSE34608 and GSE116542 databases. (B) miR-502-3p expression levels were elevated in patients with TB compared to healthy patients analyzed using the GSE116542 databases (C) miR-502-3p expression in M. tuberculosis-infected THP-1 and RAW 264.7 cells at an MOI of 10 for the indicated time was determined via reverse transcription-quantitative PCR. (D) miR-502-3p expression in M.tuberculosis-infected THP-1 and RAW 264.7 cells at indicated MOI for 48 h was determined by reverse transcription-quantitative PCR. ${ }^{*} \mathrm{P}<0.05,{ }^{* *} \mathrm{P}<0.01$ and ${ }^{* * * *} \mathrm{P}<0.001$ vs. $0 \mathrm{~h}$ or MOI of 0 . miR/miRNA, microRNA; MOI, multiplicity of infection; TB, tuberculosis; M., Mycobacterium.

transfection compared with inhibitor NC (Fig. 2A). The CFU assay demonstrated that miR-502-3p mimic transfection significantly increased the survival of M.tuberculosis from infected macrophages compared with mimic NC, whereas inhibition of miR-502-3p significantly reduced the intracellular growth of $M$. tuberculosis compared with inhibitor NC (Fig. 2B). The high number of M. tuberculosis colonies means that the phagocytosis of macrophages to M. tuberculosis is weakened and the survival of macrophages is reduced.

miR-502-3p suppresses cytokine production in $M$. tuberculosis-infected macrophages. M. tuberculosis infection promotes macrophages to produce cytokines (17). The effects of miR-502-3p on cytokines in M.tuberculosis-infected THP-1 and RAW 264.7 cells were investigated. The mRNA expression levels of IL-6, TNF- $\alpha$ and IL-1 $\beta$ were significantly induced in M.tuberculosis-infected macrophages. The mRNA expression levels of IL-6, TNF- $\alpha$ and IL-1 $\beta$, were significantly reduced in $M$. tuberculosis-infected macrophages transfected with miR-502-3p mimic, compared with the M. tuberculosis-infected only group. Moreover, downregulation of miR-502-3p significantly increased the expression of IL-6, TNF- $\alpha$ and IL-1 $\beta$, in $M$. tuberculosis-infected macrophages compared with the M. tuberculosis-infected only group (Fig. 3).

miR-502-3p directly targets ROCK1. Using TargetScan, ROCK1 was predicted to have a potential miR-502-3p target site in its 3'UTR (Fig. 4A). miR-502-3p mimic transfection significantly suppressed luciferase activity in $293 \mathrm{~T}$ cells containing the ROCK1-WT vector compared with mimic NC, whereas no change in luciferase activity was observed in cells transfected with the ROCK1-Mut vector (Fig. 4B). Western blotting demonstrated that the protein expression levels of ROCK1 were significantly increased in M. tuberculosis-infected macrophages compared with the control. Compared with the M. tuberculosis-infected only group, overexpression of miR-502-3p significantly decreased 
A

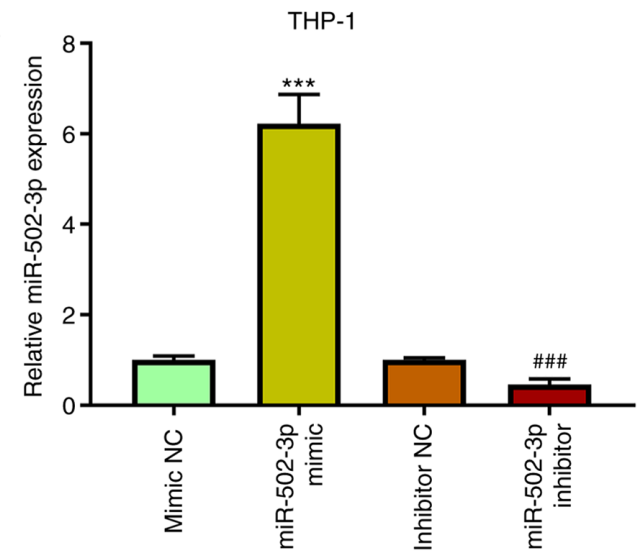

B
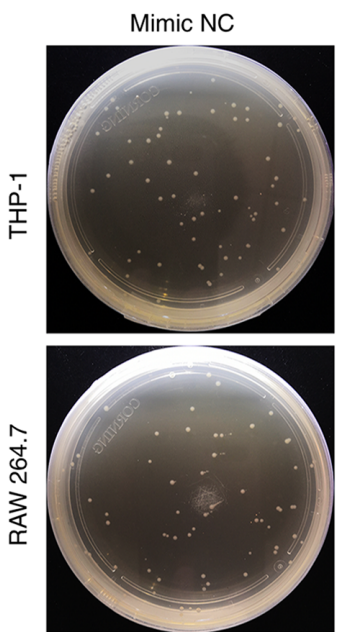

miR-502-3p mimic
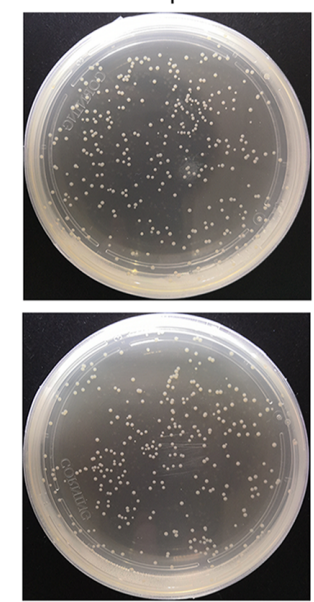

THP-1

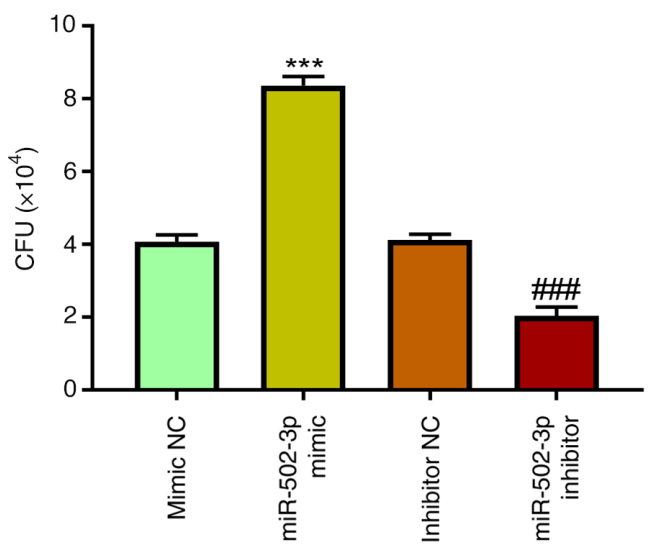

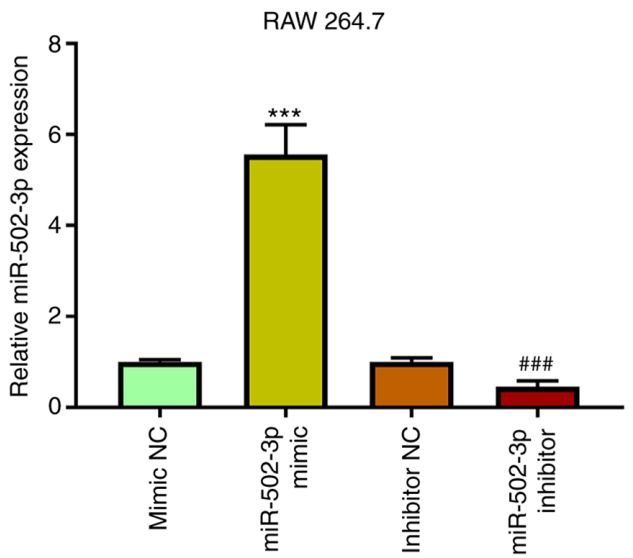

Inhibitor NC
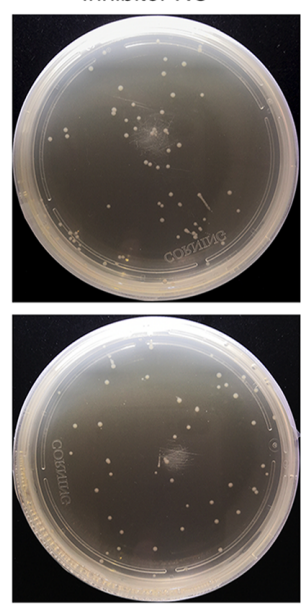

RAW 264.7

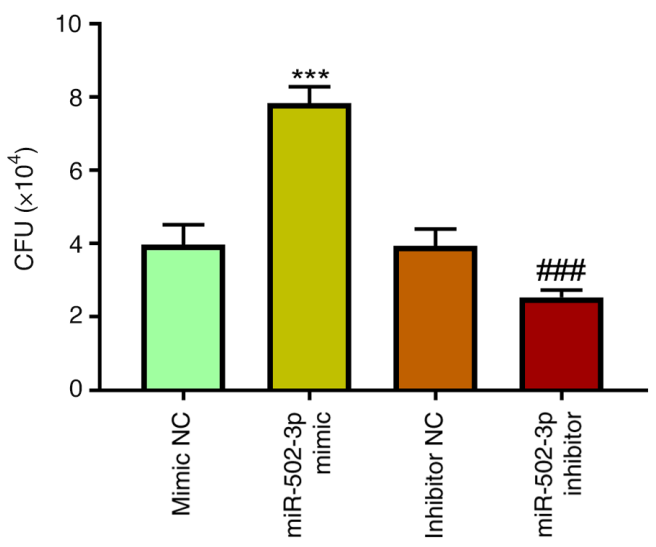

Figure 2. miR-502-3p facilitates Mycobacterium tuberculosis survival in macrophages. (A) miR-502-3p expression levels in THP-1 and RAW 264.7 cells were measured by reverse transcription-quantitative-PCR following transfection. (B) M. tuberculosis survival was measured using the colony-forming unit assay. ${ }^{* * *} \mathrm{P}<0.001$ vs. mimic NC; ${ }^{\# \#} \mathrm{P}<0.001$ vs. inhibitor NC. miR, microRNA; NC, negative control.

ROCK1 protein expression levels, whereas knockdown of miR-502-3p significantly increased ROCK1 protein expression levels (Fig. 4C).

miR-502-3p regulates the TLR4/NF- $\mathrm{B}$ signaling pathway in $M$. tuberculosis-infected macrophages. A previous study has shown that TLR-4/miR-125a/NF- $\mathrm{B}$ signaling modulates the immune response to $M$.tuberculosis infection (9). To determine the mechanism by which miR-502-3p promoted $M$. tuberculosis survival in macrophages, the TLR4/NF- $\kappa \mathrm{B}$ signaling pathway was examined. TLR4, p65 phosphorylation and IкB $\alpha$ phosphorylation were significantly increased in $M$. tuberculosis-infected THP-1 and RAW 264.7 cells. However,TLR4,p65 phosphorylation and $\mathrm{I} \kappa \mathrm{B} \alpha$ phosphorylation were significantly reduced in $M$. tuberculosis-infected THP-1 and RAW 264.7 cells transfected with miR-502-3p mimic compared with the M.tuberculosis-infected only group. Moreover, the downregulation of miR-502-3p expression significantly increased TLR4 levels and the phosphorylation of $\mathrm{p} 65$ and I $\mathrm{B} \mathrm{B} \alpha$ compared with the M.tuberculosis-infected only group (Fig. 5). Furthermore, 

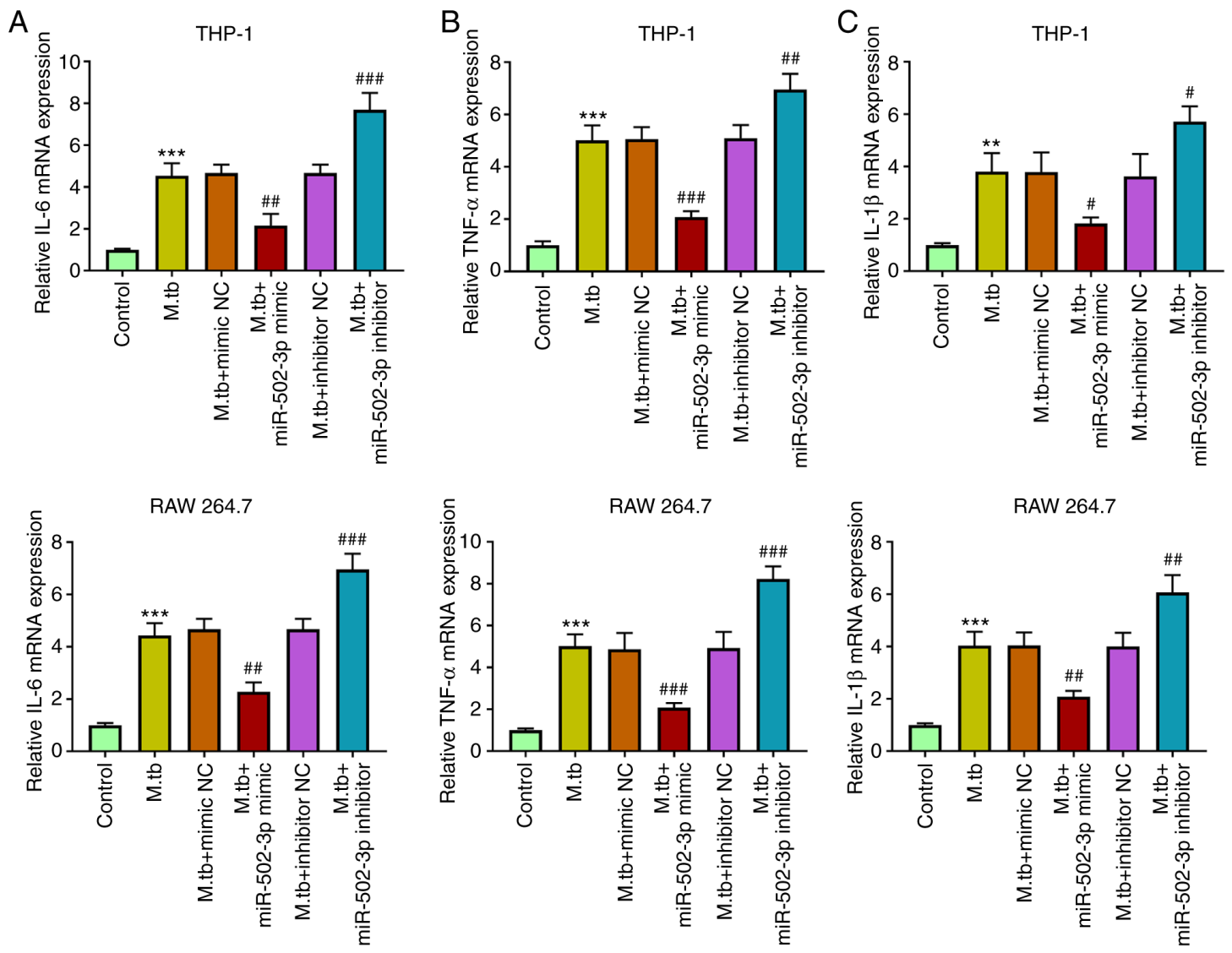

Figure 3. miR-502-3p suppresses cytokine mRNA expression levels in Mycobacterium tuberculosis-infected macrophages. (A) IL-6, (B) TNF- $\alpha$ and (C) IL-1 $\beta$ mRNA expression levels were measured by reverse transcription-quantitative PCR following transfections and infection. Untreated macrophages were used as the control. ${ }^{* *} \mathrm{P}<0.01$ and ${ }^{* * * *} \mathrm{P}<0.001$ vs. control; ${ }^{\#} \mathrm{P}<0.05,{ }^{\# \#} \mathrm{P}<0.01$ and ${ }^{\# \# \#} \mathrm{P}<0.001$ vs. M.tb. miR, microRNA; M.tb, Mycobacterium tuberculosis; NC, negative control.

compared with $M$. tuberculosis-infected only group, the expression level of p-p65 in nucleus was significantly reduced by miR-502-3p overexpression and significantly induced by miR-502-3p inhibition (Fig. 6).

ROCK1 overexpression reverses the miR-502-3p inhibitory effect on cytokine production in M. tuberculosis-infected macrophages. The effect of ROCK1 overexpression on cytokine production in miR-502-3p-overexpressing macrophages was investigated. ROCK1 overexpression was successfully achieved by transfecting THP-1 and RAW 264.7 cells with pcDNA3.1-ROCK1 (Fig. 7A). IL-6, TNF- $\alpha$ and IL-1 $\beta$ mRNA expression levels were increased in M.tuberculosis-infected THP-1 and RAW 264.7 cells. The miR-502-3p mimic increased and overexpression of ROCK1 decreased IL-6, TNF- $\alpha$ and IL-1 $\beta$ mRNA expression in M. tuberculosis-infected THP-1 and RAW 264.7 cells. Overexpression of ROCK1 partially markedly reversed the inhibitory effects of miR-502-3p mimic on IL-6, TNF- $\alpha$ and IL-1 $\beta$ levels in M. tuberculosis-infected THP-1 and RAW 264.7 cells compared with the M. tuberculosis + miR-502-3p mimic group (Fig. 7B-D).

\section{Discussion}

miRNAs have been reported to serve important roles in the host response to intracellular M. tuberculosis (18-20). For example, increased miR-20a-3p expression was shown to regulate the host immune response to promote the growth of
M. tuberculosis in human macrophages (21). In the present study, miR-502-3p expression was significantly upregulated in M. tuberculosis-infected THP-1 and RAW 264.7 cells. Furthermore, miR-502-3p overexpression significantly reduced the mRNA expression levels of inflammatory cytokines and the activation of NF- $\mathrm{kB}$ signaling through the targeting of ROCK1. miR-502-3p also serves a role in carcinomatosis, is associated with inflammation and its expression is significantly decreased following the buildup phase of venom immunotherapy (22-24). However, the role of miR-502-3p in the modulation of immune responses has not been extensively investigated.

The production of inflammatory cytokines by macrophages is regarded as a bactericidal pathway for M. tuberculosis infection (25). However, M. tuberculosis is able to resist these antimicrobial activities by establishing a niche for survival in macrophages (26). Previous studies have determined that miRNAs inhibit the expression of pro-inflammatory cytokines to generate niches favorable for mycobacterial survival $(9,27,28)$. In the present study, it was considered that the detection of mRNA changes was more direct and convenient to reflect the level of inflammatory factors. The current study demonstrated that miR-502-3p overexpression significantly reduced pro-inflammatory cytokine mRNA expression levels and promoted mycobacterial intracellular survival.

Furthermore, previous studies have demonstrated that the association between miRNAs and mRNAs serves an important role during M. tuberculosis infection. For example, miR-21-5p enhances mycobacterial survival and weakens inflammatory 


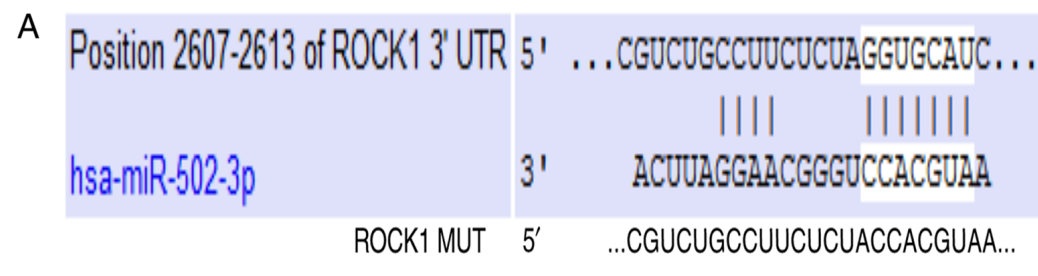

B

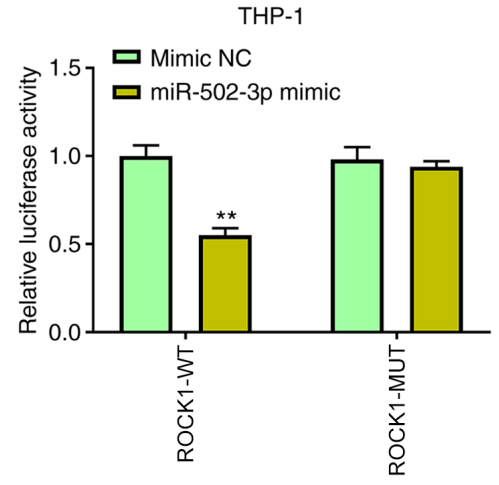

C
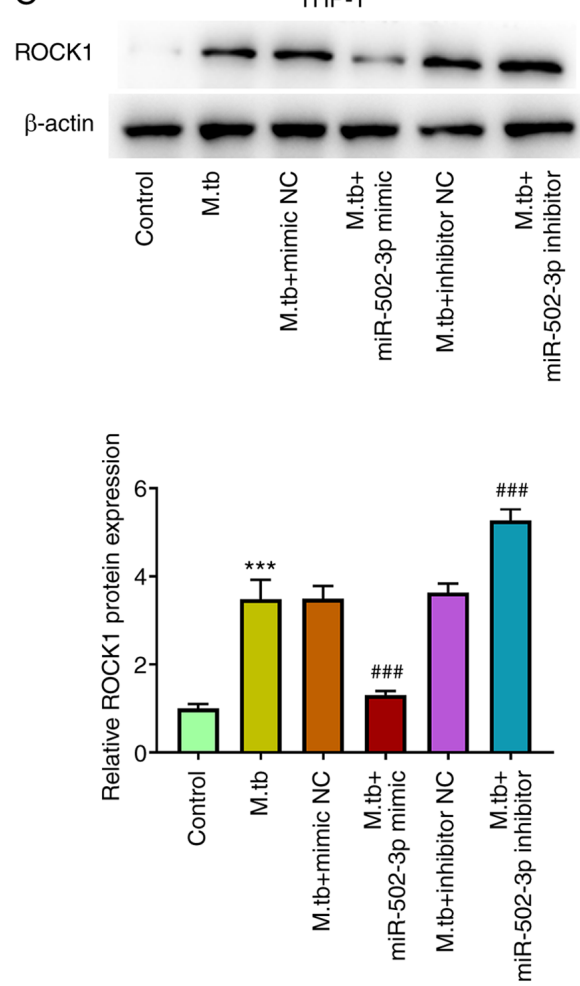

RAW 264.7
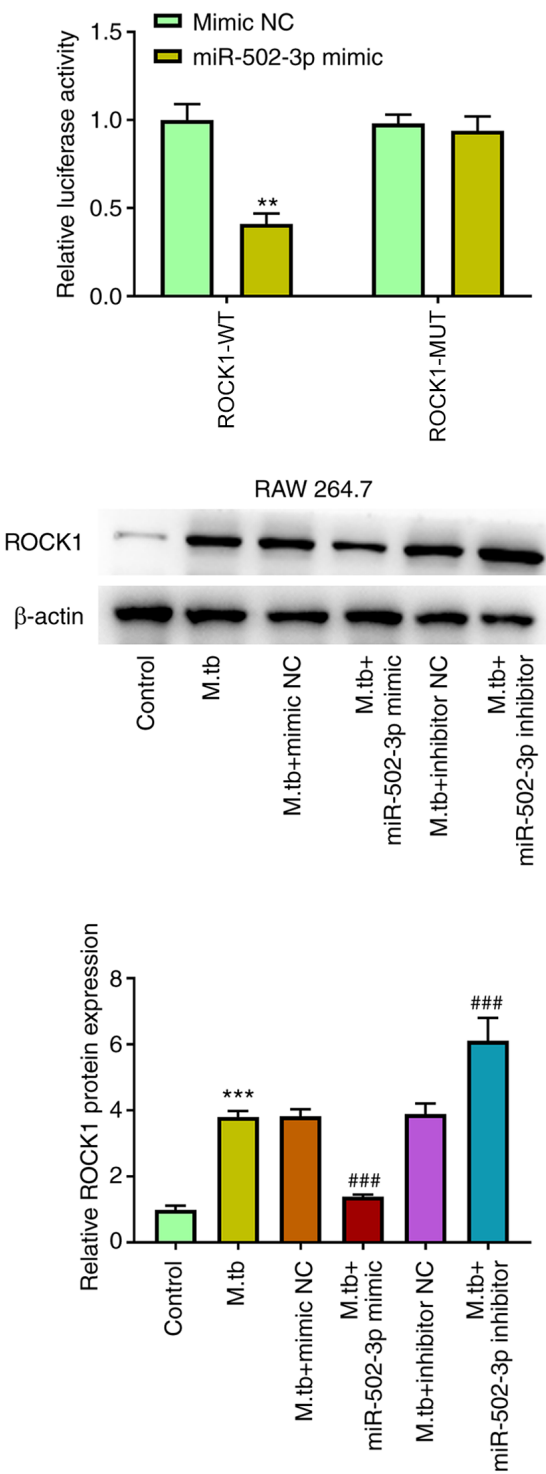

Figure 4. miR-502-3p directly targets ROCK1. (A) Predicted target site of miR-502-3p in the ROCK1 3'UTR. (B) Dual-luciferase reporter assay verified the targeting relationship between miR-502-3p and ROCK1. ${ }^{* *} \mathrm{P}<0.01$ vs. mimic NC. (C) ROCK1 protein expression levels of ROCK1 were measured by western blotting following transfections and infection. Untreated macrophages were used as the control. ${ }^{* * *} \mathrm{P}<0.001$ vs. control; ${ }^{\# \#} \mathrm{P}<0.001$ vs. M.tb. Hsa, Homo sapiens; miR, microRNA; M.tb, Mycobacterium tuberculosis; MUT, mutant; NC, negative control; ROCK1, Rho-associated coiled-coil-forming protein kinase 1; UTR, untranslated region; WT, wild-type.

responses by targeting Bcl-2 and TLR4 (29). miR-144 regulates inflammatory cytokine secretion and ERK signaling by targeting tumor progression locus 2 in $M$. tuberculosis-infected macrophages (30). In the present study, miR-502-3p overexpression promoted mycobacterial survival in macrophages by directly targeting ROCK1 to evade the immune response via the ROCK1/TLR4/NF- $\mathrm{B}$ pathway, suggesting that miR-502-3p may have a similar function as miR-21-5 during M. tuberculosis infection. Inhibition of ROCK1 alleviates lipopolysaccharide-induced inflammatory cytokine production and suppresses TLR4/NF- $\kappa \mathrm{B}$ and ERK signaling pathways in corneal epithelial cells (31). Furthermore, it has been demonstrated that an M.tuberculosis nucleoside diphosphate kinase stimulates GTPase activity of RhoA, RacI and cell division control protein 42 (32). Therefore, the RhoA/ROCK signaling pathway may be important for $M$. tuberculosis-induced inflammatory responses. The regulatory function of miRNAs through TLRs and the NF- $\mathrm{B}$ signaling pathway in M.tuberculosis infection has also recently been studied. For example, miR-148a inhibits TLR4-mediated NF- $\kappa$ B activation in 

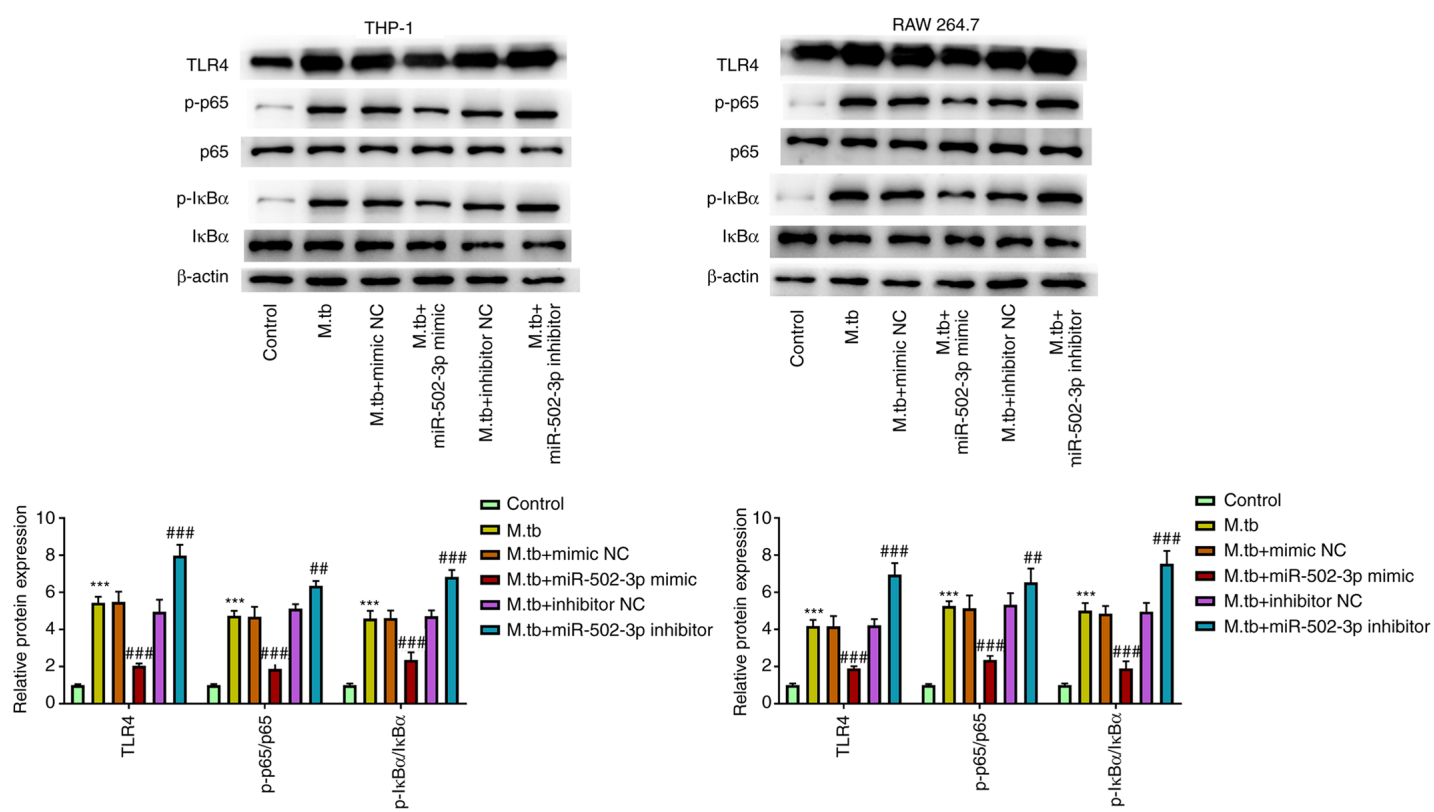

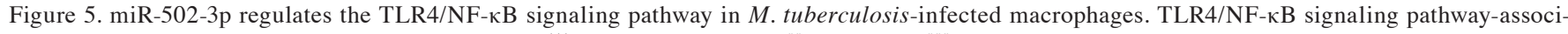
ated proteins were assessed using western blotting. ${ }^{* * *} \mathrm{P}<0.001$ vs. control; ${ }^{\# \#} \mathrm{P}<0.01$ and ${ }^{\# \# \#} \mathrm{P}<0.001$ vs. M.tb. miR, microRNA; TLR, toll-like receptor; M.tb, Mycobacterium tuberculosis; p, phosphorylated; NC, negative control.
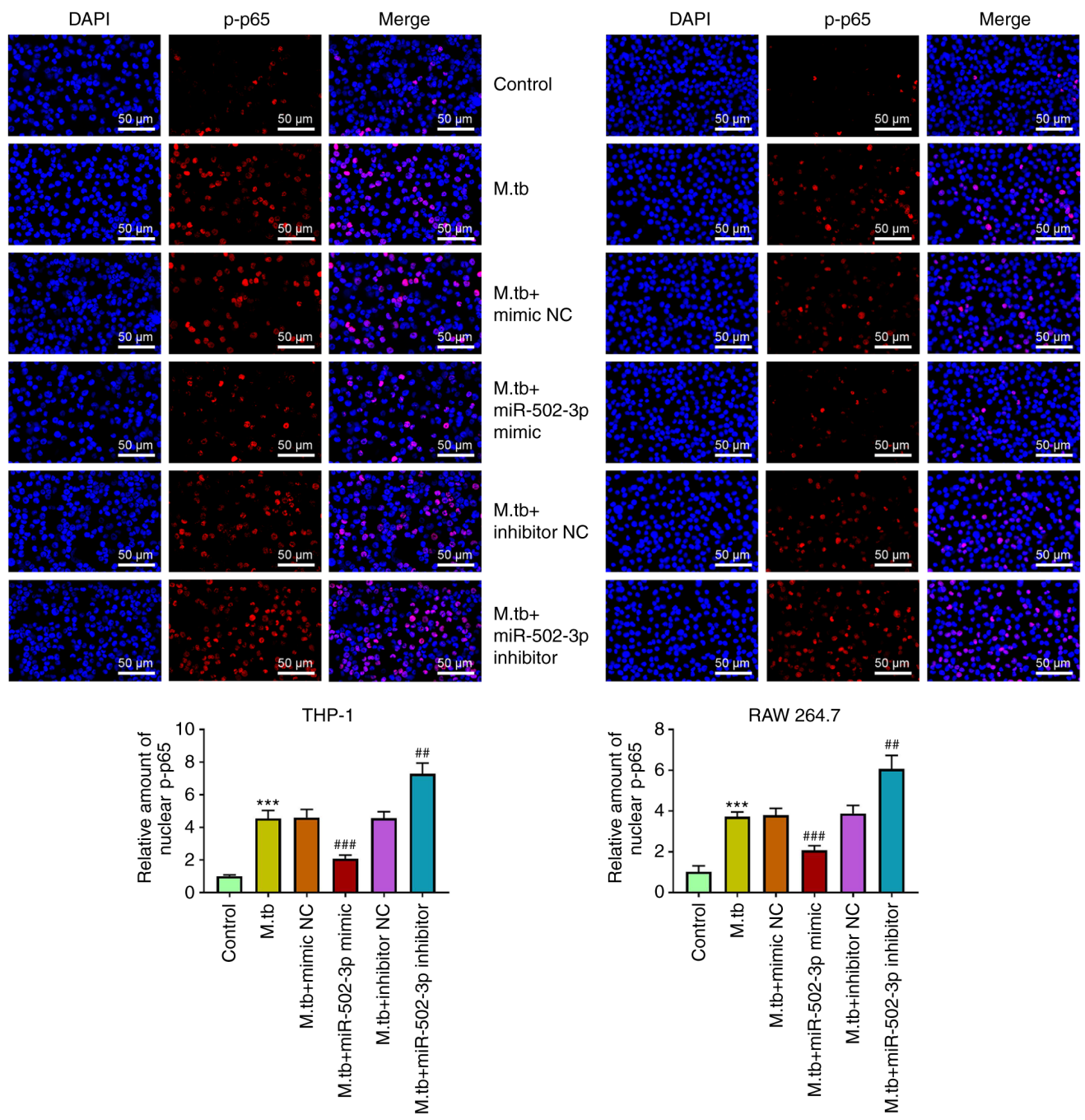

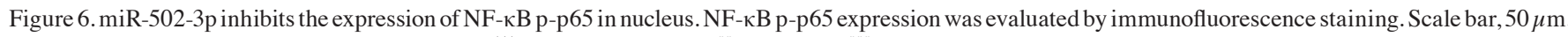
Untreated macrophages were used as the control. ${ }^{* * * *} \mathrm{P}<0.001$ vs. control; ${ }^{\# \#} \mathrm{P}<0.01$ and ${ }^{\# \# \|} \mathrm{P}<0.001$ vs. M.tb. miR, microRNA; M.tb, Mycobacterium tuberculosis; $\mathrm{NC}$, negative control. 

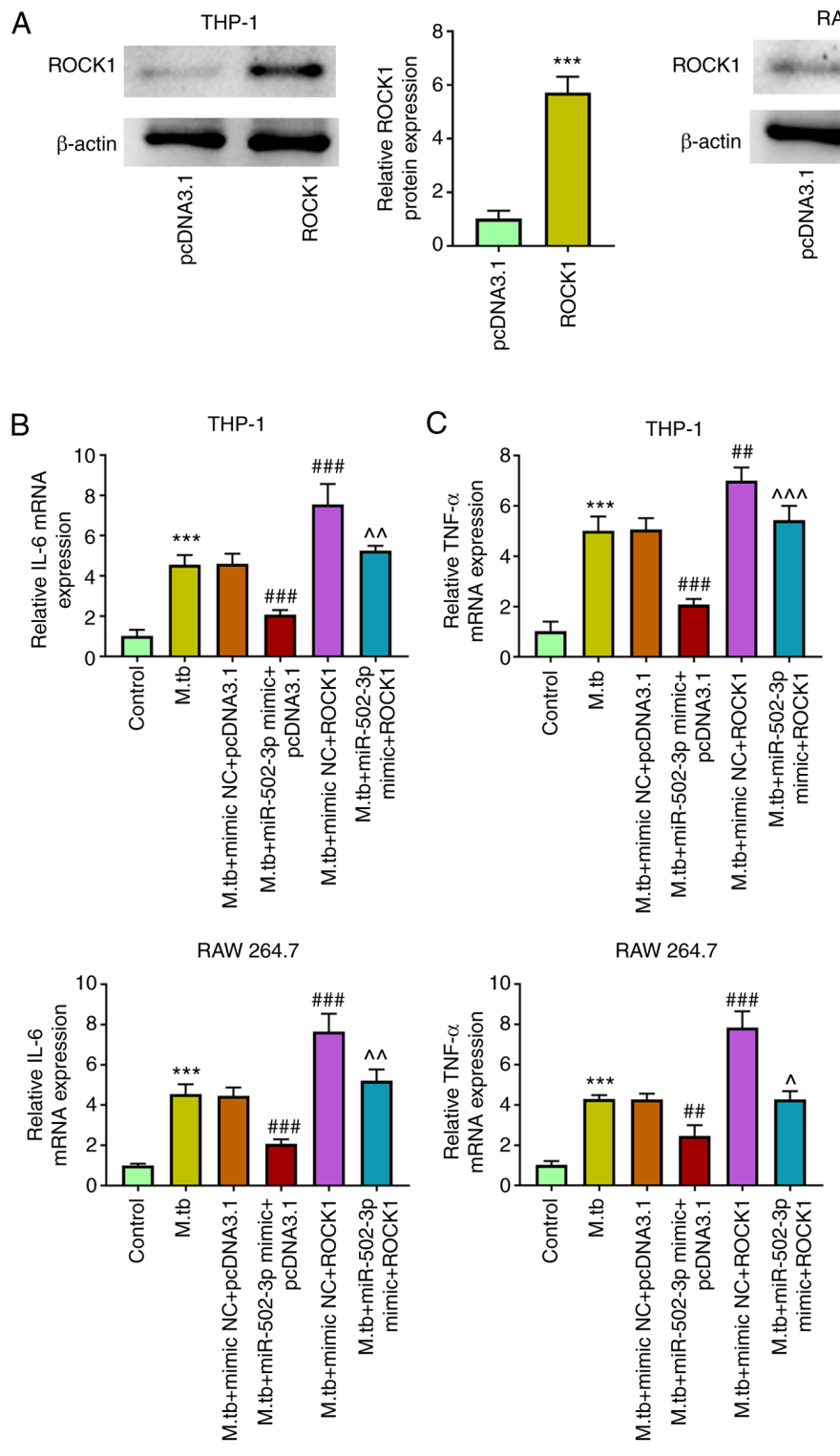
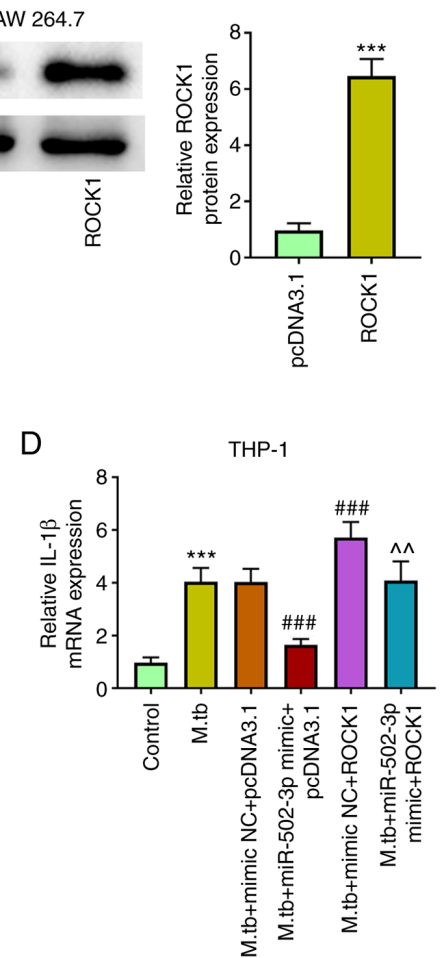

RAW 264.7

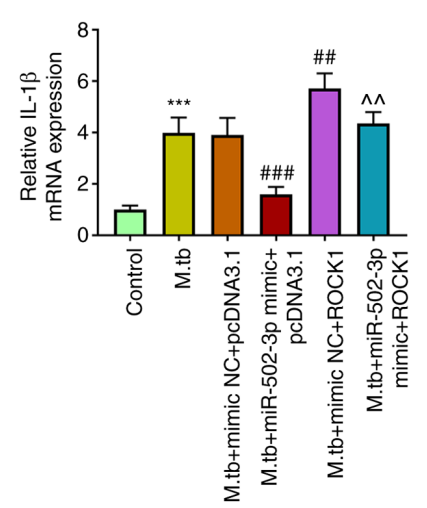

Figure 7. ROCK1 overexpression reverses the miR-502-3p inhibitory effect on cytokine mRNA expression levels in $M$. tuberculosis-infected macrophages. (A) ROCK1 protein expression levels were measured by western blotting. ${ }^{* * *} \mathrm{P}<0.001$. (B) IL-6, TNF- $\alpha$ and IL-1 $\beta$ mRNA expression levels were measured by reverse transcription-quantitative $\mathrm{PCR}$ following transfection and infection. ${ }^{* * *} \mathrm{P}<0.001$ vs. control; ${ }^{\# \#} \mathrm{P}<0.01$ and ${ }^{\# \# \#} \mathrm{P}<0.001$ vs. $\mathrm{M}$. tb; ${ }^{\wedge} \mathrm{P}<0.05,{ }^{\wedge} \mathrm{P}<0.01$ and ${ }^{\wedge \wedge} \mathrm{P}<0.001$ vs. M.tb + miR-502-3p mimic + pcDNA3.1. miR, microRNA; M.tb, Mycobacterium tuberculosis; NC, negative control; ROCK1, Rho-associated coiled-coil-forming protein kinase 1.

response to mycobacterial infection (33). The present study demonstrated that miR-502-3p regulated the host immune response to $M$. tuberculosis by directly targeting ROCK 1 to possibly attenuate TLR4-mediated $\mathrm{NF}-\kappa \mathrm{B}$ signaling pathway activation and consequent immune responses.

In conclusion, miR-502-3p promoted M. tuberculosis survival in macrophages and reduced pro-inflammatory cytokine release in $M$. tuberculosis-infected macrophages by targeting the ROCK1/TLR4/NF- $\kappa \mathrm{B}$ pathway. The expression levels of inflammatory cytokines were determined only via RT-qPCR, and the levels of these inflammatory factors were not assessed using ELISA. These results have provided the foundations for the future development of TB therapeutics.

\section{Acknowledgements}

Not applicable.

\section{Funding}

No funding was received.

\section{Availability of data and material}

The datasets used and/or analyzed during the current study are available from the corresponding author on reasonable request.

\section{Authors' contributions}

FL, YZ and ZD designed the study. YL, HY and PW performed the research and analyzed the data. FL and ZD confirmed the authenticity of all the raw data. YZ and PW wrote the paper and FL and ZD reviewed the article. All authors approved the final version of the manuscript. 


\section{Ethics approval and consent to participate}

Not applicable.

\section{Patient consent for publication}

Not applicable.

\section{Competing interests}

The authors declare that they have no competing interests.

\section{References}

1. Alzahabi KH, Usmani O, Georgiou TK, Ryan MP, Robertson BD, Tetley TD and Porter AE: Approaches to treating tuberculosis by encapsulating metal ions and anti-mycobacterial drugs utilizing nano- and microparticle technologies. Emerg Top Life Sci 4 581-600, 2020

2. Harding E: WHO global progress report on tuberculosis elimination. Lancet Respir Med 8: 30418-7, 2020.

3. Koch A and Mizrahi V: Mycobacterium tuberculosis. Trends Microbiol 26: 555-556, 2018.

4. Leopold Wager CM, Arnett E and Schlesinger LS: Mycobacterium tuberculosis and macrophage nuclear receptors: What we do and don't know. Tuberculosis (Edinb): Apr 25, 2019 (Epub ahead of print). doi: 10.1016/j.tube.2019.04.016.

5. Correia de Sousa M, Gjorgjieva M, Dolicka D, Sobolewski C and Foti M: Deciphering miRNAs' Action through miRNA Editing. Int J Mol Sci 20: 6249, 2019.

6. Zhang X, Zhu M and Hu X: Integrated miRNA and mRNA expression profiling to identify mRNA targets of dysregulated miRNAs in pulmonary tuberculosis. Epigenomics 10: 1051-1069, 2018.

7. Fu B, Xue W, Zhang H, Zhang R, Feldman K, Zhao Q, Zhang S, Shi L, Pavani KC, Nian W, et al: MicroRNA-325-3p facilitates immune escape of Mycobacterium tuberculosis through targeting LNX1 via NEK6 accumulation to promote anti-apoptotic STAT3 signaling. mBio 11: 00557-20, 2020.

8. Sahu SK, Kumar M, Chakraborty S, Banerjee SK, Kumar R, Gupta P, Jana K, Gupta UD, Ghosh Z, Kundu M and Basu J: MicroRNA 26a (miR-26a)/KLF4 and CREB-C/EBP $\beta$ regulate innate immune signaling, the polarization of macrophages and the trafficking of Mycobacterium tuberculosis to lysosomes during infection. PLoS Pathog 13: e1006410, 2017.

9. Niu W, Sun B, Li M, Cui J, Huang J and Zhang L: TLR-4/microRNA-125a/NF- $\kappa$ B signaling modulates the immune response to Mycobacterium tuberculosis infection. Cell Cycle 17: 1931-1945, 2018.

10. Chen LY, Zuraw BL, Liu FT, Huang S and Pan ZK: IL-1 receptor-associated kinase and low molecular weight GTPase RhoA signal molecules are required for bacterial lipopolysaccharide-induced cytokine gene transcription. J Immunol 169: 3934-3939, 2002.

11. Shimizu S, Tahara M, Ogata S, Hashimoto K, Morishige K, Tasaka $\mathrm{K}$ and Murata Y: Involvement of nuclear factor-kB activation through RhoA/Rho-kinase pathway in LPS-induced IL-8 production in human cervical stromal cells. Mol Hum Reprod 13: 181-187, 2007.

12. Wang ZH,Zhu D, Xie S, Deng Y,Pan Y, Ren J and Liu HG: Inhibition of Rho-kinase attenuates left ventricular remodeling caused by chronic intermittent hypoxia in rats via suppressing myocardial inflammation and apoptosis. J Cardiovasc Pharmacol 70: 102-109, 2017.

13. Juarez E, Nuñez C, Sada E, Ellner JJ, Schwander SK and Torres M: Differential expression of Toll-like receptors on human alveolar macrophages and autologous peripheral monocytes. Respir Res 11: 1465-9921, 2010.

14. Guo H, Zhang Y, Cheng BC, Fu X, Zhu P, Chen J, Chan Y, Yin C, Wang Y, Hossen M, et al: An ethanolic extract of the aerial part of Siegesbeckia orientalis L. inhibits the production of inflammatory mediators regulated by AP-1, NF- $\kappa$ B and IRF3 in LPS-stimulated RAW 264.7 cells. Biosci Trends 12: 330-337, 2018.
15. Sierra-Mondragon E, Molina-Jijon E, Namorado-Tonix C, Rodríguez-Muñoz R, Pedraza-Chaverri J and Reyes JL: All-trans retinoic acid ameliorates inflammatory response mediated by TLR4/NF- $\kappa$ B during initiation of diabetic nephropathy. J Nutr Biochem 60: 47-60, 2018.

16. Livak KJ and Schmittgen TD: Analysis of relative gene expression data using real-time quantitative PCR and the 2(-Delta Delta C(T)) method. Methods 25: 402-408, 2001.

17. Korbel DS, Schneider BE and Schaible UE: Innate immunity in tuberculosis: Myths and truth. Microbes Infect 10: 995-1004, 2008.

18. Sharma N, Verma R, Kumawat KL, Basu A and Singh SK: miR-146a suppresses cellular immune response during Japanese encephalitis virus JaOArS982 strain infection in human microglial cells. J Neuroinflammation 12: 30, 2015.

19. Pachathundikandi SK and Backert S: Helicobacter pylori controls NLRP3 expression by regulating hsa-miR-223-3p and IL-10 in cultured and primary human immune cells. Innate Immun 24 : $11-23,2018$

20. Yu T, Ju Z, Luo M, Hu R, Teng Y, Xie L, Zhong C, Chen L, Hou W, Xiong Y and Feng Y: Elevated expression of miR-146a correlates with high levels of immune cell exhaustion markers and suppresses cellular immune function in chronic HIV-1-infected patients. Sci Rep 9: 18829, 2019.

21. Cui J, Li Z, Cui K, Gao Y, Zhang B, Niu J and Wang Y: MicroRNA-20a-3p regulates the host immune response to facilitate the Mycobacterium tuberculosis infection by targeting IKK $\beta / \mathrm{NF}-\kappa \mathrm{B}$ pathway. Int Immunopharmacol 91: 107286, 2021.

22. Jin H, Yu M, Lin Y, Hou B, Wu Z, Li Z and Sun J: MiR-502-3P suppresses cell proliferation, migration, and invasion in hepatocellular carcinoma by targeting SET. Onco Targets Ther 9: 3281-3289, 2016

23. Zhang J, Hou L, Liang R, Chen X, Zhang R, Chen W and Zhu J: CircDLST promotes the tumorigenesis and metastasis of gastric cancer by sponging miR-502-5p and activating the NRAS/MEK1/ERK1/2 signaling. Mol Cancer 18: 80, 2019.

24. Specjalski K, Maciejewska A, Pawłowski R, Chełmińska M and Jassem E: Changes in the expression of microRNA in the buildup phase of wasp venom immunotherapy: A pilot study. Int Arch Allergy Immunol 170: 97-100, 2016.

25. Lv J, He X, Wang H, Wang Z, Kelly GT, Wang X, Chen Y, Wang T and Qian Z: TLR4-NOX2 axis regulates the phagocytosis and killing of Mycobacterium tuberculosis by macrophages. BMC Pulm Med 17: 194, 2017.

26. Shi L, Jiang Q, Bushkin Y, Subbian S and Tyagi S: Biphasic dynamics of macrophage immunometabolism during Mycobacterium tuberculosis infection. mBio 10: e2550-18, 2019.

27. Li X, Huang S, Yu T, Liang G, Liu H, Pu D and Peng N: MIR-140 modulates the inflammatory responses of Mycobacterium tuberculosis-infected macrophages by targeting TRAF6. J Cell Mol Med 23: 5642-5653, 2019.

28. Gu X, Gao Y,Mu DG and Fu EQ: MiR-23a-5p modulates mycobacterial survival and autophagy during Mycobacterium tuberculosis

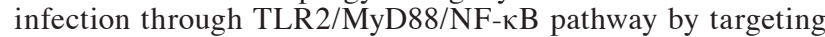
TLR2. Exp Cell Res 354: 71-77, 2017.

29. Zhao Z, Hao J, Li X, Chen Y and Qi X: MiR-21-5p regulates mycobacterial survival and inflammatory responses by targeting Bcl-2 and TLR4 in Mycobacterium tuberculosis-infected macrophages. FEBS Lett 593: 1326-1335, 2019.

30. Liu HY: Down-regulation of miR-144 after Mycobacterium tuberculosis infection promotes inflammatory factor secretion from macrophages through the Tpl2/ERK pathway. Cell Mol Biol 62: 87-93, 2016

31. Gong J, Guan L, Tian P, Li C and Zhang Y: Rho kinase type 1 (ROCK1) promotes lipopolysaccharide-induced inflammation in corneal epithelial cells by activating toll-like receptor 4 (TLR4)-mediated signaling. Med Sci Monit 24: 3514-3523, 2018.

32. Chopra P, Koduri H, Singh R, Koul A, Ghildiyal M, Sharma K, Tyagi AK and Singh Y: Nucleoside diphosphate kinase of Mycobacterium tuberculosis acts as GTPase-activating protein for Rho-GTPases. FEBS Lett 571: 212-216, 2004.

33. Wu H, Bao Y, Wang L, Li X and Sun J: Mycobacterium marinum down-regulates miR-148a in macrophages in an Esx A-dependent manner. Int Immunopharmacol 73: 41-48, 2019.

This work is licensed under a Creative Commons Attribution-NonCommercial-NoDerivatives 4.0 International (CC BY-NC-ND 4.0) License. 